\title{
GCU
}

Glasgow Caledonian

University

University for the Common Good

\section{Parental injustice appraisals in the context of child pain: examining the construct and criterion validity of the IEQ-Pc and IEQ-Ps}

Baert, Fleur; Miller, Megan Marie; Trost, Zina; Hirsh, Adam Todd; McParland, Joanna; De Schryver, Maarten; Vervoort, Tine

Published in:

Journal of Pain

DOI:

10.1016/j.jpain.2019.06.012

Publication date:

2020

Document Version

Author accepted manuscript

Link to publication in ResearchOnline

Citation for published version (Harvard):

Baert, F, Miller, MM, Trost, Z, Hirsh, AT, McParland, J, De Schryver, M \& Vervoort, T 2020, 'Parental injustice appraisals in the context of child pain: examining the construct and criterion validity of the IEQ-Pc and IEQ-Ps', Journal of Pain, vol. 21, no. 1-2, pp. 195-211. https://doi.org/10.1016/j.jpain.2019.06.012

\section{General rights}

Copyright and moral rights for the publications made accessible in the public portal are retained by the authors and/or other copyright owners and it is a condition of accessing publications that users recognise and abide by the legal requirements associated with these rights.

Take down policy

If you believe that this document breaches copyright please view our takedown policy at https://edshare.gcu.ac.uk/id/eprint/5179 for details

of how to contact us. 
Parental injustice appraisals in the context of child pain: Examining the construct and criterion validity of the IEQ-Pc and IEQ-Ps.

Baert, F. ${ }^{1}$, Miller, M. M. ${ }^{2}$, Trost, Z. ${ }^{3}$, Hirsh, A. T. ${ }^{2}$, McParland, J. ${ }^{4}$, De Schryver, M. ${ }^{1}$ \& Vervoort, ${ }^{1}{ }^{1}$

${ }^{1}$ Department of Experimental-Clinical and Health Psychology, Ghent University, Belgium

${ }^{2}$ Indiana University - Purdue University Indianapolis, Department of Psychology

${ }^{3}$ University of Alabama at Birmingham, Department of Psychology

${ }^{4}$ Glasgow Caledonian University, Department of Psychology

Corresponding author: Fleur Baert, Department of Experimental-Clinical and Health Psychology, Ghent University, Henri Dunantlaan 2, 9000 Ghent, Belgium.

Electronic mail may be sent to Fleur.Baert@,UGent.be

Disclosures: see below

Funding: this work was financially supported by an IASP collaborative grant awarded to Tine Vervoort.

Conflict of interest statement: there are no conflicts of interest that may arise as a result of the research presented in this article.

Keywords: children, parents, chronic pain, perceived injustice, catastrophizing, emotion 


\begin{abstract}
A growing pediatric and adult literature highlights the role of injustice appraisals in adjustment to pain. However, interpersonal injustice dynamics have remained largely unexplored. The present study investigated the factor structure and criterion validity of parentally-adjusted versions of the Injustice Experience Questionnaire, assessing childoriented (IEQ-Pc) and self-oriented appraisals (IEQ-Ps) in the context of child pain. Participants were triads of healthy children $\left(\mathrm{N}=407, \mathrm{M}_{\mathrm{age}}=12\right)$ and both their parents and dyads of children with chronic pain $\left(\mathrm{N}=319, \mathrm{M}_{\mathrm{age}}=14\right)$ and one parent. In both samples, children completed measures of functional disability and quality of life (physical, emotional, social, academic); parents completed the IEQ-Pc, IEQ-Ps, and a measure of parental catastrophizing about child pain. Across samples, a confirmatory oblique two-factor model (Severity/Irreparability-Blame/Unfairness) provided a better fit to the data compared to a onefactor model; nevertheless, the two-factor solution was considered suboptimal. A post-hoc exploratory factor analysis consistently revealed one factor. In terms of criterion validity, the IEQ-Pc and IEQ-Ps demonstrated differential associations depending on the child's pain vs. healthy status, independent of parental catastrophizing. Further, findings in the healthy sample indicated that fathers' self-oriented injustice appraisals related to lower child social function. In the clinical sample, parental child-oriented injustice appraisals related to greater child functional disability and lower physical, emotional, social, and academic function. Current findings support the unique role of parental injustice appraisals, assessed by the IEQ-Pc and IEQ-Ps, in understanding child pain, but also suggest these may only partially capture the phenomenology of parental injustice appraisals in the context of child pain.
\end{abstract}




\section{Perspective}

This manuscript presents an examination of the construct and criterion validity of two parentally adjusted versions of the Injustice Experience Questionnaire. These measures could be valuable tools for clinicians in examining how parents respond to their child's pain as it impacts both the child's life as well as the parents'. 


\section{Introduction}

Research among adult and pediatric samples suggests that pain-related injustice appraisal - an appraisal comprising elements of severity and irreparability of pain-related loss, as well as perceived blame and unfairness ${ }^{[50]}$ - contribute significantly and uniquely to adverse pain outcomes ${ }^{[34,47,50,51,53,54]}$. However, despite continuing advances, research has been largely restricted to an intrapersonal perspective, utilizing the Injustice Experience Questionnaire (IEQ ${ }^{[49]}$ ) to examine individuals' (in)justice appraisals regarding personal pain.

According to recent literature, pain arouses essential appraisal processes both among those experiencing it and those observing another's pain ${ }^{[9,20,36,38]}$. These interpersonal dynamics are highlighted by studies indicating parental pain-related appraisals significantly impact child pain experience ${ }^{[18,19,35]}$. Indeed, there is a robust link between parental catastrophic appraisals about child pain and deleterious child outcomes, including greater pain, disability, and school absence ${ }^{[19]}$. In this respect, parental appraisals of injustice regarding child pain may likewise inform child outcomes. Parenting a child with chronic pain can entail witnessing the child experience pain-related losses and goal disruption (e.g., diminished social or academic participation $)^{[12,29,30,37,38,39]}$, as well as frustration at inadequate pain-control efforts ${ }^{[4,5]}$. Recent findings by Miller and colleagues ${ }^{[35]}$ offer preliminary support for the impact of parents' pain-related injustice appraisal on child pain outcomes.

Importantly, parenting a child with chronic pain may engender substantial challenges, losses, and goal interference for parents themselves. Indeed, parents of children with chronic pain report greater emotional distress and limitations in social and professional functioning ${ }^{[29,30,37,39]}$. Accordingly, parenting a child with pain may foster appraisals of injustice oriented toward the child's pain experience (e.g. "It is not fair that my child is enduring this") as well as toward one's own experience as a parent (e.g., "It's not fair that I as a parent have to endure this"). To date, only one study ${ }^{[35]}$ has examined parental injustice 
appraisals in the context of their child's pain, using a parent version of the IEQ. This study offered initial insight regarding the importance of parents' child-oriented injustice appraisals in understanding child pain outcomes, yet some critical questions remain unaddressed. Most importantly, the validity of the parentally adjusted IEQ, whereby items were minimally rephrased to reflect parental appraisals of their child's pain rather than personal pain, was not assessed. This is critically important, since the extrapolation of measures to different contexts is a common practice in psychological assessment (e.g., PCS, PCS-C, PCS-P, PCS-S ${ }^{[10,19,52]}$ ). However, when doing so, context-specific content informing the operationalization of the construct must be taken into consideration, and validity must be established before continuing to use the measure in further research. Furthermore, the notion of parental self-oriented appraisals and their impact upon child outcomes have yet to be examined.

As such, the current study sought to examine the utility and validity of two parentallyadjusted IEQ instruments differentially targeting child- and self-oriented injustice appraisals in response to child pain - the IEQ-Pc and IEQ-Ps, respectively. Study 1 examined their factor structure (i.e., per Sullivan et $\mathrm{al}^{[50]}$ ) in a sample of parents of healthy children. Study 2 subsequently tested this factor structure among parents of children with chronic pain. Both studies also examined criterion validity of both measures in relation to child pain-related outcomes. In line with findings that caregiver appraisals are particularly detrimental to child outcomes when arising from perceived violation of self- rather than child-oriented goals $^{[18,59,60]}$, we expected parents' self-oriented injustice appraisals to be more disadvantageous for child outcomes. Further, to assess the unique role of parental injustice appraisals, current analyses controlled for parental catastrophizing about child pain ${ }^{[34,50,51,54]}$. Comparisons between samples allowed for examination of the role of child pain status (i.e., healthy children versus children with chronic pain) in parental self and child-oriented injustice appraisals and their relationship to child pain-related outcomes. 


\section{Study 1 - Injustice appraisal among parents of healthy children}

\subsection{Method}

\subsubsection{Participants}

Participants were 407 triads of healthy school children and both their mother and father, recruited via nine Flemish primary and secondary schools. A total of 2,612 children and their parents were invited to take part; 2,261 children and 1,006 parents (549 mothers, 457 fathers, 613 families total) completed the study between March and May 2016. Current analyses only include triads (i.e., mother, father, child) for whom complete self-report data were available.

The final sample consisted of 407 triads of children (189 boys and 215 girls, mean age $=12$ years, $S D=2.09, M I N=8, M A X=16)$ and both their mothers and fathers. All children attended school fulltime; one child's age was not recorded. Mean age was 42 years $(S D=4.51)$ for mothers $(\mathrm{N}=176)$ and 44 years $(S D=5.23)$ for fathers $(\mathrm{N}=176)$. The majority of parents were either married or cohabiting (89.4\%) and had completed a higher education $(77.5 \%$ of mothers, $65.6 \%$ of fathers). Missing data analysis revealed that $1 \%$ of child questionnaire scores and $1 \%$ of parent questionnaire scores were not computed, as more than $25 \%$ of questionnaire items was not completed.

\subsubsection{Parent measures}

Parental Appraisals of Injustice regarding Child Pain. Parental appraisals of injustice regarding their child's pain experience were assessed with the parentally-adjusted version of the IEQ ${ }^{[50]}$ as used previously by Miller and colleagues ${ }^{[35]}$ - the IEQ-Parent About Child or IEQ-Pc. Adjustment involved minimal rephrasing of the items to reflect a parental perspective focusing on the child experiencing pain, rather than personal pain. The item content and response format remained unchanged. The IEQ-Pc included 12 items and asked parents to rate the extent to which they experience 12 feelings/thoughts such as "My child should not have to 
live this way" and "I can't believe this has happened to my child" specifically in reference to their child's general pain experience (i.e., when thinking about their child pain experience). Parents were not instructed to reflect on any specific type of pain. Items are rated on a Likert scale ranging from 0 ("never") to 4 ("all the time") and responses are summed to calculate a total score. Higher scores indicate higher child-oriented injustice perception. Parallel to the original IEQ, the 12 items are thought to constitute 2 subscales labeled "Severity/Irreparability of loss" and "Blame/Unfairness". This parental version of the IEQ has been described elsewhere ${ }^{[35]}$. For the purposes of the current study, the IEQ-Pc was translated and back translated with language-specific adjustments into Dutch, following standard procedures ${ }^{[3]}$. The original IEQ measure regarding personal pain experience has been validated in adult $^{[31,42,50]}$ and child ${ }^{[34]}$ chronic pain samples. For the current study the IEQ-Pc demonstrated high internal consistency $(\alpha=.93)$.

Parental Appraisal of Injustice regarding the Self. Parental appraisals of injustice regarding their own experience as a consequence of their child's pain were assessed using similar IEQ adaptation - the IEQ-Parent About Self or IEQ-Ps. Accordingly, item content and response frame also remained unchanged but items were now rephrased to reflect a parental perspective focusing on the parent's situation caring for a child experiencing pain. The IEQPs instructed parents to reflect specifically on how their child's pain affected their life and to rate 12 items such as "It all seems so unfair" and "Because of my child's pain, my life will never be the same" using a 0 ("never") to 4 ("all the time") scale. Higher summed scores again indicated greater self-oriented injustice perception in the context of child pain. As with the IEQ-Pc, translation occurred from an existing English-language version, and was likewise considered to reflect "Severity/Irreparability of loss" and "Blame/Unfairness" subscales. The language-adapted version demonstrated high internal consistency $(\alpha=.94)$ in the present study. 
Parental Pain Catastrophizing. The Pain Catastrophizing Scale for Parents ${ }^{[19]}$ (PCP-P) was administered to assess parental catastrophic thinking about their child's pain. This measure is an adaptation of the adult Pain Catastrophizing Scale ${ }^{[52]}$ (PCS) and the Pain Catastrophizing Scale for Children ${ }^{[10]}$ (PCS-C). The PCS-P consists of 13 items, describing various thoughts and feelings parents may experience when their child is in pain. Items reflect subscales of rumination (e.g., "When my child is in pain, I can't keep it out of my mind"), magnification ("When my child is in pain, I keep thinking of other painful events"), and helplessness ("When my child is in pain, it's terrible and I think it's never going to get better"). Parents rate each item on a 5-point scale, ranging from 0 ("not at all") to 4 ("extremely"), reflecting the extent to which they experience each of the thoughts and feelings when their child is in pain. The Dutch version of the PCS-P has been validated in both clinical and non-clinical parent populations ${ }^{[10]}$. The PCS-P demonstrated high internal consistency $(\alpha$ $=.92)$ in the present study.

\subsubsection{Child measures}

Functional Disability. Child function was assessed by means of the Functional Disability Inventory $^{[62]}$ (FDI), a self-report measure for children and adolescents with a variety of pediatric conditions. The FDI asks participants to rate the difficulty with which they carry out 15 daily activities (e.g. "walking up stairs") on a 5-point scale ranging from 0 ("No trouble") to 4 ("Impossible"). Higher levels of functional disability are indicative of increasing difficulty in age-appropriate physical and psychosocial functioning due to physical health status. The English version of the FDI has been validated in a sample of pediatric pain patients $^{[8]}$. The Dutch translation has been utilized in various studies ${ }^{[10,19]}$ and showed high internal consistency $(\alpha=.90)$ in the present study.

Health-related Quality of Life. The Generic Core Scales of the Pediatric Quality of Life Inventory $4.0^{[56]}$ (PedsQL) assessed health-related quality of life as reported by the child. 
These scales encompass Physical functioning ( 8 items; e.g. "It's hard for me to run"), Emotional functioning (5 items; e.g. "I feel anxious or scared"), Social functioning (5 items; e.g. "Other children don't want to be my friend"), and Academic functioning (5 items; e.g. "I easily forget things"). All twenty-three items are rated on a 4-point scale, ranging from 0 ("Never") to 4 (“Almost always"). Items are reverse scored to a 0-100 scale, with a higher score being indicative of higher health-related quality of life. The PedsQL has been validated in a Dutch sample by Engelen and colleagues ${ }^{[13]}$ and yielded adequate internal consistency ( $\alpha$ $=.68-.82)$ in the current study.

\subsubsection{Procedure}

For participant recruitment, schools were sent a letter and later contacted by telephone by the researchers. Schools consenting to participate were asked to distribute information briefs among children in the $3^{\text {rd }}$ to $9^{\text {th }}$ grade, who were asked to provide these briefs, and enclosed consent to parents. Parents could explicitly declare refusal (on behalf of themselves and their child) to partake in the research. Children whose parents did not explicitly refuse participation completed the child measures during regular school hours, after which they were given a set of parent questionnaires for mothers and fathers. All studies were approved by the Psychology and Educational Sciences Ethical Committee.

\subsubsection{Statistical analyses}

All analyses (with the exception of regression analyses) were conducted separately for the IEQ-Pc and IEQ-Ps, as well as for mothers and fathers. To examine the construct validity of the IEQ-Pc and IEQ-Ps, their factor structure was assessed by means of confirmatory factor analysis (CFA), using the statistical program $\mathrm{R}$ (Lavaan package). CFA is the preferred statistical approach since 1) there is substantial empirical evidence for a priori specifying the number and patterns of common factors as reflected by studies examining the factor structure of the IEQ in a variety of different samples - across age groups, clinical conditions and 
countries $^{[31,34,42,50]}$ and 2) items of the IEQ-Pc and IEQ-Ps were only minimally rephrased to refer to parental perceptions of child pain rather than personal pain. Based on available literature $^{[31,34,42,50]}$, we first examined an oblique two-factor model (Model 2) in both measures, assuming that 6 "Severity/Irreparability of loss" items and 6 "Blame/Unfairness" items reflect two correlated dimensions. However, given the fact that, across both IEQ-Pc and IEQ-Pcs, the above factors showed a significantly higher correlation $(r=.95-.98)$ than observed in previous psychometric studies $(r=.45-.50)^{[31,34,42,50]}$, a one-factor model (Model 1) was also examined for both measures, in which all 12 items were assumed to be indicative of a single latent factor of "perceived injustice". Goodness-of-fit indices were evaluated for both models by means of the maximum likelihood algorithm. In line with Jackson, Gillaspy and Purc-Stephenson ${ }^{[28]}$, several fit indices were used to assess goodness-of-fit for both models. A non-significant $\chi^{2}$ indicated an acceptable fit to the observed data. However, this index is influenced by sample size. To address this problem, the $\chi^{2} /$ degrees of freedom (CMIN/DF ratio), which represents the minimum sample discrepancy divided by degrees of freedom ${ }^{[64]}$ was also checked. Ratios as low as 2 or as high as 5 were considered indicative of a reasonable fit ${ }^{[26,27]}$. Root Mean Square Error of Approximation (RMSEA) values below .06 were considered a reasonable fit ${ }^{[26,27]}$. For the Comparative Fit Index (CFI), values above .90 were considered as indicative of an adequate fit to the data ${ }^{[26,27]}$. The fit of both models was compared by means of the chi-square difference statistic. A significant p-value for this test indicates the larger model provides a better fit to the data ${ }^{[26]}$. However, since CFA demonstrated a suboptimal fit of the tested factor-structure across samples, post-hoc exploratory factor analysis (EFA) was performed to allow for a more profound understanding of the data (i.e., a potentially different number of factors or items that would show insufficient factor loadings or cross loadings). EFA was conducted using promax rotation and the maximum likelihood estimation method. Parallel analysis was performed to identify the 
number of factors as this is considered the most reliable method, carrying the least risk for overfactoring and subjectivity ${ }^{[21,44]}$. Descriptive and regression analyses were also performed, in order to assess other dimensions of validity that are likewise important in evaluating assessment instruments.

Paired samples t-tests were used to assess differences between mothers' and fathers' injustice appraisals and between levels of parental child- and self-oriented appraisals. Independent samples t-tests were computed to examine potential differences in parental child and self-oriented injustice appraisal depending on the sex of their child.

To examine criterion validity of the IEQ-Pc and IEQ-Ps measures, hierarchical linear regression analysis was performed to examine the relationships among parental child- and self-oriented injustice appraisals and child pain outcomes (i.e., functional disability and physical, emotional, social, and academic functioning). IEQ-Pc and IEQ-Ps total scores were entered as independent variables within all regression analyses. This was justified given the high correlation between subscales (see above) and since analyses utilizing subscale scores yielded similar results. In all regression analyses, child age and sex (boys coded "0", girls coded "1") were entered in a first step to control for sociodemographic variables. Considering the significant correlation between appraisals of injustice and pain catastrophizing observed in previous studies $^{[31,34,42,50]}$ as well as the current study $(r=.49-.51$, all $p<.01$; see Table 2$)$, parental catastrophizing about child pain was entered as a control variable in the second step, allowing to examine the unique role of parental child- and self-oriented injustice appraisals. In the last step, both the IEQ-Pc and IEQ-Ps measures were entered for mothers and fathers separately. As the IEQ-Ps and IEQ-Pc were highly correlated $(r=.71-.79)$, Variance Inflation Factors (VIF) were examined for each regression analysis; these proved to be within acceptable range suggesting no problems with multicollinearity (VIF $=1.0-2.9$ ). Correction for multiple comparisons was not considered appropriate as the current study did not meet any of 
the conditions required for this adjustment (i.e., a) a universal null hypothesis of interest, b) the same test is repeated in many subsamples and c) searching for significant associations without a priori hypotheses for multiple tests ${ }^{[40]}$ ). All analyses were performed separately for mothers and fathers.

\subsection{Results}

\subsubsection{Confirmatory factor analyses}

\subsubsection{Mothers}

\section{- INSERT TABLE 1 ABOUT HERE -}

Appraisals of Injustice regarding Child Pain (IEQ-PC). Table 1 presents goodness-offit indices for the different models tested for both the IEQ-Pc and IEQ-Ps, as well as the chisquare difference statistic for each comparison of model fit. For IEQ-Pc data provided by mothers in the sample, the two-factor model provided a better fit relative to the one-factor model (i.e., $\chi_{\text {diff }}^{2}=6.69, p<.001$ ), but was nevertheless considered suboptimal. Specifically, adequate fit was observed for the Comparative Fit Index $(\mathrm{CFI}=.92)$, but not for CMIN/DF ratio $(\mathrm{CMIN} / \mathrm{DF}=5.50)$ and Root Mean Square Error of Approximation $(\mathrm{RMSEA}=.106)$. Further, a high correlation was observed between the "Severity/Irreparability" "Blame/Unfairness" dimensions of the two-factor model $(r=.97)$.

Appraisals of Injustice regarding the Self (IEQ-PS). For IEQ-Ps, the two-factor model provided a better, but still suboptimal fit to mothers' (i.e. $\chi_{\text {diff }}^{2}=32.80, p<.001$; CMIN/DF=7.48; RMSEA=.128; CFI=.91) self-oriented appraisal data. Parallel to childoriented appraisal findings above, a high correlation was noted between the "Severity/Irreparability" "Blame/Unfairness" dimensions of the two-factor model $(r=.95)$

\section{- INSERT FIGURE 1 ABOUT HERE -}

\subsubsection{Fathers}


Appraisals of Injustice regarding Child Pain (IEQ-PC). Relative to the one-factor solution, the two-factor model did not provide a better fit to father IEQ-Pc data (i.e., $\chi^{2}$ diff $=$ 3.27, NS). Goodness-of-fit was again evaluated as suboptimal, yielding an acceptable CMIN/DF ratio and CFI of 4.77 and .94 respectively, yet an unacceptable RMSEA value of .097. Again, a high correlation was observed between both factors $(r=.97)$.

Appraisals of Injustice regarding the Self (IEQ-PS). For fathers' self-oriented injustice data, the two-factor model also provided a suboptimal fit (i.e. $\chi^{2}$ diff $=19.54, p<.001$; $\mathrm{CMIN} / \mathrm{DF}=6.20$; RMSEA=.115; CFI=.93). Also, a high correlation was observed between both factors in the model $(r=.98)$.

\section{- INSERT FIGURE 2 ABOUT HERE -}

\subsubsection{Post-hoc exploratory factor analysis}

Exploratory factor analysis (EFA) demonstrated similar results for both mother and father data and for both the IEQ-Pc and IEQ-Ps. In particular, findings indicated that, across analyses, all items were moderately to strongly correlated ( $r=.25$ to .81$)$ and showed acceptable loadings (i.e., greater than .40; ranging between .46 and .90). Parallel analysis consistently suggested a one-factor solution, further challenging the validity of the original two-factor structure in this particular context. While the observed suboptimal fit suggests that caution is needed as both the IEQ-Ps and IEQ-Pc may not fully capture the construct of parental injustice appraisals, findings derived from CFA and post-hoc EFA justify the inclusion of all items of each respective measure into one total score to be used in further analyses to examine measures' criterion validity.

\subsubsection{Descriptive statistics and correlation analyses}

Mean scores, standard deviations, Cronbach's alphas, and correlation coefficients for all parent and child measures are summarized in Table 2. Parents' self-reported levels of catastrophizing about their child's pain were similar to findings reported in previous studies in 
samples of healthy school children ${ }^{[10]}$. Children also reported similar levels of functional disability and health-related quality of life to other samples of healthy school children $^{[28,29,30,31]}$. Both mothers and fathers reported significantly higher levels of childoriented injustice appraisals (IEQ-Pc scores) compared to self-oriented injustice appraisals $($ IEQ-Ps scores) $-t(396)=5.83, p<.001$ and $t(392)=7.01, p<.001$ for mothers and fathers, respectively. Fathers reported significantly higher levels of child-oriented injustice appraisals than mothers $(t(394)=-1.98 ; p<.05)$. No significant difference emerged between mothers and fathers' self-oriented injustice appraisals $(t(391)=-.73$, ns $)$. One-way ANOVA revealed no significant difference in child- or self-oriented injustice appraisal by fathers ( $F_{\text {child- }}$ oriented $\left.(3,394)=1.191, \mathrm{~ns} ; F_{\text {self-oriented }}(3,391)=1.220, \mathrm{~ns}\right)$ or in child-oriented injustice appraisal by mothers $\left(F_{\text {child-oriented }}(3,396)=1.461, \mathrm{~ns}\right)$ depending on the sex of the child. However, mothers of boys did report significantly higher levels of self-oriented injustice than did mothers of girls $\left(F_{\text {self-oriented }}(3,394)=3.763, p<.05\right)$.

A significant correlation was observed between IEQ-Pc and IEQ-Ps scores across both mothers and fathers $(r=.71-.79, p<.01)$. In line with previous research examining injustice appraisals regarding personal pain ${ }^{[34,50,51]}$, a significant correlation was found between the PCS-P and both the IEQ-Pc and IEQ-Ps $(r=.42-.51, p<.01)$. Of particular interest for the present study, correlation analyses indicated that both fathers' IEQ-Pc and IEQ-Ps scores correlated significantly with child emotional $(r=-.16, p<.01)$ and social functioning; $(r=-.20$, $p<.01)$ indicating higher levels of father-reported child- and self-oriented injustice appraisals were associated with lower levels of child-reported emotional and social functioning in the current sample. No significant correlations were observed between mothers' IEQ-Pc and IEQPs scores child outcomes (all $r \leq .10, \mathrm{~ns}$ ).

\section{- INSERT TABLE 2 ABOUT HERE -}

\subsubsection{Regression analyses}


Table 3 displays results for a series of multiple hierarchical regression analyses examining the role of either mother or father child- and self-oriented injustice appraisals in explaining child functional disability and the 4 dimensions of health-related quality of life (i.e., child physical, emotional, social and academic functioning). Below we first report on results of regression analyses for mothers and then for fathers.

\subsubsection{Mother-reported child- and self-oriented injustice appraisals}

Child functional disability. Analyses with child-reported functional disability as a dependent variable revealed only a significant association with the child's age, with older children reporting lower levels of functional disability $(\beta=-.15, p<.01)$. No significant main effects were observed for child sex $(\beta=.01, \mathrm{~ns})$, mothers' catastrophizing about child pain $(\beta=$ $.03, \mathrm{~ns})$ or either the IEQ-Pc $(\beta=.03, \mathrm{~ns})$ or IEQ-Ps $(\beta=.02, \mathrm{~ns})$ measures.

Child physical functioning. Regression analyses revealed only a significant association with child age $(\beta=.12, p<.05)$, indicating that older children reported higher levels of child physical functioning. No other significant effects were observed (all $|\beta|$ values $<.10, \mathrm{~ns})$

Child emotional functioning. Analyses revealed a significant association with child $\operatorname{sex}(\beta=-.14, p<.01)$ indicating girls reported lower levels of emotional functioning than boys. No other significant effects were observed (all $|\beta|$ values $<.10$, ns).

Child social functioning. Analyses with child-reported social functioning as a dependent variable revealed no significant associations with child sex, age or parental catastrophizing about child pain (all $|\beta|$ values $<.09$, ns). Further, no significant association with mothers' injustice perceptions was found (all $|\beta|$ values $<.11$, ns).

Child academic functioning. Analyses revealed a significant association with child age $(\beta=-.11, p<.5)$, indicating that older children reported lower levels of academic 
functioning. No significant associations with child sex, parental catastrophizing about child pain or parental injustice perceptions were found (all $|\beta|$ values $<.11$ ).

\subsubsection{Father-reported child- and self-oriented injustice appraisals}

Child functional disability. Regression analysis with child functional disability as a dependent variable revealed only a significant association with child age $(\beta=-.16, p<.01)$, indicating that older children reported lower levels of functional disability. No other significant associations were observed (all $|\beta|$ values $<.09$, ns).

Child physical functioning. Analysis again only revealed a significant association with child age $(\beta=.14, p<.01)$, suggesting that older children reported higher levels of physical functioning. Child sex, fathers' catastrophizing and both injustice measures were not significant (all $|\beta|$ values $<.09$, ns).

Child emotional functioning. Analysis with child emotional functioning as a dependent variable yielded a significant association with child $\operatorname{sex}(\beta=-.13, p<.05)$, indicating that girls reported lower levels of emotional functioning than boys. No other significant associations were observed (all $|\beta|$ values $<.12$, ns).

Child social functioning. Regression analysis revealed a significant association with fathers' self-oriented injustice $(\beta=-.17, p<.05)$, indicating that higher fathers' IEQ-Ps scores were associated with lower levels of child social functioning. No other significant associations were found (all $|\beta|$ values $<.01$, ns)

Child academic functioning. Analyses with child academic functioning as a dependent variable revealed no significant associations with any of the included predictors (all $|\beta|$ values $<.1, \mathrm{~ns})$.

\subsection{Summary of findings (Study 1$)$}


The aim of Study 1 was to investigate the factor structure and criterion validity of the IEQ-Pc (parental child-oriented injustice appraisal) and the IEQ-Ps (parental self-oriented injustice appraisal) in the context of child pain within a sample of healthy children and their parents. With the exception of fathers' IEQ-Ps data, confirmatory factor analysis did indicate that a two-factor model (i.e., a "Severity/Irreparability" factor and "Blame/Unfairness" factor) fitted the data better than did a one-factor model. However, no solid evidence was obtained for construct validity of either measure as the fit of this two-factor model was consistently evaluated as suboptimal with a high correlation across factors being observed for both mothers and fathers. Post-hoc EFA consistently suggested a one factor structure and showed moderate to strong correlations between all items, thereby justifying the use of the IEQ-Pc and IEQ-Ps total score in further regression analyses. Findings further indicated that both mothers and fathers reported significantly higher levels of child-oriented injustice appraisal than selforiented injustice appraisal. Finally, results of regression analysis indicated that, after controlling for child sex, age, and paternal catastrophizing about child pain, fathers' injustice appraisal was significantly associated with child-reported social functioning, with higher levels of injustice appraisal relating to lower levels of child social functioning, hence providing some support for the criterion validity and unique value of paternal injustice appraisals in understanding deleterious child outcomes. Notably, this effect was only obtained for fathers' self- not child-oriented injustice appraisals, suggesting that paternal self-oriented injustice appraisals may be more disadvantageous for child outcomes. This suggests that parental child- and self-oriented injustice appraisals may differentially affect child pain outcomes. No effects were observed for mother-reported child- and self-oriented injustice appraisals.

\section{Study 2 - Injustice appraisal among parents of children with chronic pain}

\subsection{Method}




\subsubsection{Participants}

Participants were children and adolescents and one of their parents presenting to an American Midwestern pediatric pain management clinic at a children's hospital located in a large urban setting between January 2014 and March 2017. Patients were referred to the clinic by primary care providers or specialists due to persistent pain related to chronic disease, injury, sports activity, or surgery. The clinic provides interdisciplinary services to patients including anesthesiology, physical therapy, nutrition, and psychology. Patients and one of their parents filled out a battery of measures during their initial and follow-up clinic appointments. In total, 319 children (90 boys, 229 girls) and 319 parents took part in the study. Unlike Study 1, no assessment was made of whether the partaking parent was the mother or father of the child. Therefore, analyses in the clinical sample could not be run separately for mothers and fathers. Mean age of participating children and adolescents in this sample was 14 years $(S D=3.01$, range 8.4-22.5 years $)$. All children and adolescents attended school fulltime. Age of the participating parent was not registered. Children reported an average pain intensity of 4.48 (i.e., on a scale ranging from 0 to $10 ; S D=2.75$ ). Mean pain intensity at the time of data collection was 4.45 (i.e., on a scale from 0 to $10 ; S D=2.76$ ). Most frequently reported pain locations included abdominal pain, limb pain, and back pain.

\subsubsection{Measures}

Parallel to Study 1, parental child- and self-oriented appraisals of injustice and catastrophizing about their child's pain were assessed by means of the IEQ-Pc, IEQ-Ps, and PCS-P, respectively, yielding good internal consistencies with Cronbach's alphas ranging between $.90-.94$. As noted, the child-oriented injustice measure (IEQ-Pc) has been described in a previous study ${ }^{[35]}$. The IEQ-Ps reflected the same modification toward parents' appraisal of injustice regarding the self as described in Study 1. Child functional disability and healthrelated quality of life were indexed by means of the same measures as described in the method 
section of Study 1. Average pain intensity was assessed by means of a Numerical Rating Scale (NRS-11) ranging from 0 ("no pain at all”) to 10 ("most pain ever"). All child report measures yielded a good internal consistency in the current study with $\alpha=.90$ for the FDI and $\alpha=.83-.88$ for the PEDS-QL subscales. Missing data analysis revealed that $8 \%$ of child questionnaire scores and $12 \%$ of parent questionnaire scores were not computed, as more than $25 \%$ of questionnaire items were not completed.

\subsubsection{Procedure}

Data for this study was obtained using a retrospective review of clinical data. Patients and their participating parent completed forms electronically at the beginning and, if necessary, end of their clinic appointment as a routine part of the clinic's pain assessment program. Prior to conducting analyses, all data were anonymized by clinic personnel to ensure compliance with the Health Insurance Portability and Accountability Act (1996) and Protected Health Information standards. All study procedures were approved by the Indiana University Institutional Review Board. The current investigation is part of a larger study protocol. Prior studies by Miller and colleagues ${ }^{[34,35]}$ have included portions of the current dataset. However, research questions assessed in the current study are unique and different from previously published work.

\subsubsection{Statistical analysis}

As in Study 1, all analyses (with the exception of regression analyses) were performed separately for both injustice measures. As noted, analyses do not include distinction between mothers and fathers. The factor structure of the IEQ-Pc and IEQ-Ps was examined in the current clinical sample by means of confirmatory factor analysis, including the same one- and two-factor models as in Study 1. Again, a post-hoc exploratory factor analysis was performed with Promax rotation and maximum likelihood estimation method. Parallel analysis was applied as the preferred method for reliable factor retention ${ }^{[21,44]}$. 
Independent samples t-test was performed to examine differences between samples in terms of level of injustice appraisal as well as differences between parental injustice appraisals depending on sex of the child ${ }^{[17]}$. A significant difference in age was observed between samples $\left(M_{\text {healthy }}=11.8, S D_{\text {healthy }}=.10 ; M_{\text {clinical }}=14.31, S D_{\text {clinical }}=.17 ; t(723)=13.00\right.$, $p<.001$ ), where the mean age of children was higher in the clinical sample. Further, the boygirl ratio differed significantly between samples $\left(\chi^{2}(1)=25.38, p<001\right)$. The clinical sample showed a significantly greater proportion of girls, whereas the non-clinical sample showed a more even distribution. However, regression analyses consistently controlled for child age and sex, in order to account for the influence of these potential confounding factors ${ }^{[15]}$.

Hierarchical linear regression analysis was again performed to investigate the impact of parental child- and self-oriented injustice appraisals on child pain-related outcomes. In a first step, child age, sex (boys coded "0", girls coded "1) and average pain intensity were entered. As was the case in Study 1, parental catastrophizing about child pain was entered in a second step. In the last step, child- and self-oriented injustice were entered simultaneously. As both injustice measures again showed a high correlation within the clinical sample $(r=.73)$, Variance Inflation Factors were examined for each regression analysis to check for multicollinearity; these proved to be within acceptable range (VIF $=1.0-2.8$ ). As mentioned in the method section of Study 1, Bonferroni correction for multiple comparisons was not performed, as the current study did not meet its required conditions ${ }^{[40]}$.

\subsection{Results}

\subsubsection{Confirmatory factor analyses}

Appraisals of Injustice regarding Child Pain (IEQ-Pc). Goodness of fit indices for the different models tested in both samples are presented in Table 1. The two-factor model provided a better, yet suboptimal fit to the data $\left(\chi_{\text {diff }}^{2}=125.74, p<0.001\right)$ than the one-factor model. The two-factor model demonstrated an acceptable fit based on Comparative Fit Index 
$(\mathrm{CFI}=.91)$ and $\mathrm{CMIN} / \mathrm{DF}$ ratio $(\mathrm{CMIN} / \mathrm{DF}=4.08)$. However, an RMSEA value of .099 was an indication of inadequate fit. A high correlation was also observed between both factors $(r=.96)$

Appraisals of Injustice regarding Self (IEQ-PS). Again, the two-factor model provided a better fit to the data $\left(\chi_{\text {diff }}^{2}=15.886, p<.001\right)$. However, goodness of fit indices again proved to be suboptimal with only CFI demonstrating acceptable fit $(\mathrm{CFI}=.91)$. The CMIN/DF ratio and RMSEA did not demonstrate acceptable fit $(\mathrm{CMIN} / \mathrm{DF}=5.24 ; \mathrm{RMSEA}=.115)$. Both factors of the self-oriented injustice measure were also highly correlated $(r=.96)$.

- INSERT FIGURE 3 ABOUT HERE -

\subsubsection{Post-hoc exploratory factor analysis}

Parallel to the results of Study 1, exploratory factor analysis (EFA) demonstrated similar results for both measures. Across analyses, all items were correlated $(r=.10-.74)$ and showed acceptable loadings (i.e., greater than .40; ranging between .42 and .86). Parallel analysis again suggested a one-factor solution for both measures, further challenging the validity of the original two-factor structure in this particular context. Findings derived from CFA and post-hoc EFA justify the inclusion of all items of each respective measure into one total score to be used in further analyses.

\subsubsection{Descriptive statistics and correlation analysis}

Descriptive statistics and Cronbach's alphas for parent and child self-report measures are presented in Table 4. Parents reported significantly higher levels of both child- and selforiented injustice appraisal than parents in the school sample $(t(1116)=-11.84, p<.00)$ and $(t(1106)=-37.92, p<.001)$ for IEQ-Pc and IEQ-Ps, respectively. Similar to parents in the school sample, parents in the clinical sample reported significantly higher levels of childoriented injustice than self-oriented injustice $(t(312)=-27.58, p<.001)$. Further, relative to healthy children in Study 1, children in Study 2 reported significantly higher levels of 
functional disability $(t(72)=-16.53, \mathrm{p}<.001)$ and significantly lower levels of health-related quality of life $\left(t(672)_{\text {physical functioning }}=24.03, p<.001 ; \mathrm{t}(675)_{\text {emotional functioning }}=7.25, p<.001\right.$; $\left.t(675)_{\text {social functioning }}=8.21, p<.001 ; t(672)_{\text {academic functioning }}=18.86, p<.001\right)$. Children in Study 2 reported levels of functional disability and health-related quality of life (PedsQL) that were comparable with other pediatric chronic pain samples ${ }^{[9,10]}$. No significant difference was observed in levels of either parental child- or self-oriented injustice appraisals depending on child sex $\left(F_{\text {child-oriented }}(1,311)=.008\right.$, ns; $F_{\text {self-oriented }}(1,317)=2.263$, ns $)$. Significant Pearson correlations between parental child- and self-oriented injustice appraisal and all measured child outcomes were found in the expected direction, with correlation coefficients ranging between -.32 and .31 (all ps <.05). Specifically, findings indicated that higher levels of parental self- and child-oriented injustice appraisals were associated with lower levels of child physical, emotional, social and academic functioning and higher levels of child functional disability.

\section{- INSERT TABLE 4 ABOUT HERE -}

\subsubsection{Regression analysis}

Table 5 displays results for a series of hierarchical regression analyses examining the multivariate relationships between parental child- and self-oriented injustice appraisals and child functional disability and the 4 dimensions of health-related quality of life (i.e., physical, emotional, social and academic functioning).

Child functional disability. Analyses with child functional disability as dependent variable revealed significant associations with child $\operatorname{sex}(\beta=.12, \mathrm{p}<.05)$ and pain intensity $(\beta=.39, p<.001)$, indicating that girls and children with higher pain intensity reported higher functional disability. No significant associations with child age, parental catastrophizing, and parental self-oriented injustice appraisals were observed (all $|\beta|$ values $<1.37$, ns). 
Child physical functioning. Analyses revealed that younger children $(\beta=.14, p<.05)$, girls $(\beta=-.16, p<.05)$, and children reporting higher levels of average pain intensity $(\beta=-.34$, $p<.001)$ reported worse physical functioning. The association with parental child-oriented injustice appraisals was also significant $(\beta=-.23, p<.05)$, as higher IEQ-Pc scores were associated with worse physical functioning in the child. Parental catastrophizing and selforiented injustice appraisals did not relate significantly to child physical functioning (all $|\beta|$ values $<.03, \mathrm{~ns})$.

Child emotional functioning. A significant association with average pain intensity $(\beta=-.21, \quad p<.001)$ and parental child-oriented injustice appraisals $(\beta=-.23, \quad p<.05)$ was observed, indicating that higher levels of child average pain intensity and IEQ-Pc scores were associated with worse emotional functioning reported by the child. No significant relations with child age, sex, parental catastrophizing, or self-oriented injustice appraisal were observed (all $|\beta|$ values $<.13$, ns).

Child social functioning. A significant effect for parental child-oriented injustice appraisals $(\beta=-.29, p<.01)$ reflected that higher IEQ-Pc scores were associated with worse social functioning in the child. No significant associations with child age, sex, pain intensity, parental catastrophizing, or self-oriented injustice appraisal were observed (all $|\beta|$ values $<.15$, ns).

Child academic functioning. Significant relations with child age $(\beta=.08, p<.05)$ and average pain intensity $(\beta=-.26, p<.05)$ indicated that younger children and those reporting higher average pain intensity reported lower levels of academic functioning. Parental childoriented injustice appraisals were again significant $(\beta=-.29, p<.01)$, with higher IEQ-Pc scores being associated with worse academic functioning reported by the child. No significant association was observed for child sex, parental catastrophizing about pain, or parental selforiented injustice appraisal (all $|\beta|$ values $<.07$, ns). 


\section{- INSERT TABLE 5 ABOUT HERE -}

\subsubsection{Summary of findings (Study 2)}

The aim of Study 2 was to investigate the factor structure and criterion validity of the parental child- and self-oriented versions of the IEQ in the context of child chronic pain and examined within a clinical child sample. In line with findings from Study 1, confirmatory factor analysis consistently showed a better fit of the two-factor model (i.e., Severity/Irreparability and Blame/Unfairness) to both child- and self-oriented injustice appraisal data; however, goodness of fit was considered suboptimal for both measures. A high correlation was also found between the two factors for both measures. Results of post-hoc EFA consistently suggested a one factor structure and moderate to strong correlations between all items. Parallel to Study 1, parents in the clinical sample reported higher levels of childoriented injustice appraisals compared to self-oriented appraisals. Findings also further attested to the importance of parental injustice appraisals in understanding child pain-related outcomes. However, in contrast to Study 1, findings of Study 2 indicated that higher levels of child- rather than self-oriented injustice appraisals were associated with worse functional disability and health-related quality of life (i.e., physical, emotional, social and academic functioning) among children in the sample. This suggests that the differential impact of parental child- and self-oriented injustice appraisals may be dependent on child pain status. Of further interest, parents in Study 2 reported higher levels of both child- and self-oriented injustice appraisals than did parents in Study 1. This finding supports the notion that pediatric chronic pain creates additional challenges, losses, blame and other conditions associated with higher levels of injustice appraisals. ${ }^{[29,30]}$

\section{General Discussion}

The present study sought to investigate the factor structure and criterion validity of childand self-oriented versions of a new, Parental Injustice Experience Questionnaire - the IEQ-Pc 
and IEQ-Ps. Two questionnaire studies were conducted, first in a sample of healthy school children and their parents and subsequently in a sample of children with chronic pain and their parents. The findings can be summarized as follows. In terms of factor structure, findings of the confirmatory analyses for both the IEQ-Pc and IEQ-Ps - with the exception of fathers' child-oriented appraisal data in the healthy sample - indicated that an oblique two-factor model provided a better fit to the data compared to a one-factor model. However, contrary to expectations and prior psychometric studies of the original $\operatorname{IEQ}^{[1,31,34,42,50]}$, the two-factor solution did not achieve adequate fit. Findings across samples also indicated a strong positive correlation between both factors that was remarkably higher than correlations previously observed. In line with this finding, post-hoc exploratory factor analysis consistently supported a one-factor solution, further challenging the two-dimensional structure of parental injustice appraisals in this context. All items yielded acceptable loadings on a single factor, justifying the use of one total score for each measure when examining their explanatory value in understanding child outcomes.

In terms of criterion validity, higher parental injustice appraisals were related to worse child outcomes across samples. However, important distinctions were observed for parental child- and self-oriented injustice appraisals depending on child pain status and - for the nonclinical sample that included both parents - between mothers' and fathers' injustice appraisals. Specifically, findings from the clinical sample indicated that parents' childoriented injustice appraisals were significantly and uniquely related to worse child pain outcomes (i.e., functional disability as well as physical, social, emotional, and academic function). Within the healthy sample, fathers' but not mothers' self-oriented injustice appraisals were negatively associated with child social function. Further, while parents of children with chronic pain reported higher levels of both child- and self-oriented injustice 
appraisal compared to parents of healthy children, parents in both samples endorsed higher levels of child- versus self-oriented injustice cognition.

The current findings extend previous research in a number of key areas. Most importantly, factor analytic findings warn against extrapolating measures to different contexts by minimal adaptation of instructions and item phrasing. Rather, these findings attest to the importance of establishing a measure's validity in the context of interest. As such, it is possible that the IEQ-Pc and IEQ-Ps only partially reflect the construct of parental injustice appraisals about child pain, leaving some context-specific content unaddressed. However, considering the above reservations, the current study also demonstrated that parental injustice appraisals regarding the child or the self - assessed by means of the IEQ-Pc and IEQ-Ps in their current form - do relate significantly and uniquely with child functioning, thus substantiating preliminary evidence by Miller and colleagues ${ }^{[35]}$. Further, differential findings across clinical and nonclinical samples provide new insight regarding the context-specificity of parental injustice appraisals regarding child pain. Specifically, in line with expectations, parents in the clinical sample reported significantly higher levels of child- and self-oriented injustice.

The present findings also provide preliminary support for the value of assessing both parents' child- and self-oriented injustice appraisals in future research and clinical assessment. As noted, both self- and child-oriented injustice appraisals were significantly higher among parents in the clinical sample compared to the healthy sample, attesting both to parents' recognition of child suffering as well as acknowledgement of the personally-disruptive impact of parenting a child in pain. This aligns with literature that consistently documents the psychosocial and functional challenges associated with caring for a child with chronic pain ${ }^{[29,30,37]}$. Furthermore, while not directly assessed by the current investigation, a recent study of caregivers for adult chronic musculoskeletal pain patients found higher caregiving demands to 
be significantly associated with higher injustice appraisals regarding one's role as caregiver $^{[36]}$.

A theoretical account of the distinction between self- and child-oriented injustice appraisal is offered by an affective-motivational model of interpersonal pain dynamics recently proposed by Vervoort and Trost ${ }^{[59]}$. The model posits that observing another in pain creates a fundamental tension between goals observers hold for the individual in pain (i.e., other-oriented goals) and those they hold for themselves (i.e., self-oriented goals). Prioritization of self- versus other-oriented goals may contribute to differential emotional and motivational processes that in turn impact the nature and effectiveness of caregiving behavior. While optimal caregiving behavior is thought to arise from prioritization of other-oriented goals, flexible attunement to self- vs. other-oriented goals is likewise considered critical to effective care.

Arguably, while child-oriented goals foster caregiving behavior and attunement to the child's needs, evidence suggests that an excessive or enduring focus on child pain (or childoriented injustice appraisals) can likewise be detrimental. For instance, persistent parental attempts to control child pain and associated protective responses (e.g., to shield the child from aversive/unjust experiences), have been observed to have a negative effect on child coping with chronic pain ${ }^{[4,5,6,58]}$. Further, chronic neglect or frustration of parental selforiented goals may undermine parental well-being, thereby indirectly hindering effective caregiving ${ }^{[60,63,66]}$. Future research may address whether such explanation accounts for the differential findings regarding self- vs. child-oriented injustice appraisals amongst parents in the healthy and clinical samples.

Additionally, further research will allow better understanding of potential cognitiveaffective mechanisms underlying these effects. For instance, depending on the extent to which parents are affected by child's pain (e.g., emotionally, functionally, socially,...), parental 
injustice appraisals may be more pronounced and increasingly impact child functioning. Negative emotional responses such as anger, stemming from the violation of child- and selforiented goals, may be particularly relevant in this context ${ }^{[47]}$. Heightened parental attention to child pain cues or towards parental anger cues may be another mechanism to be explored ${ }^{[51]}$. Further, broader justice literature suggests that justice-related information communicated through others' emotional cues (e.g., anger, guilt) can influence observers' judgments and associated responses ${ }^{[23,55]}$. In this respect, it is possible that child emotional expression may differentially arouse child- or self-oriented injustice appraisals in parents.

Findings within the non-clinical sample cautiously suggest that these processes may be particularly pronounced for fathers. Specifically, fathers demonstrated higher scores for both child- and self-oriented injustice appraisals, and only fathers' self-oriented injustice appraisals were associated with child functioning. To date, few studies ${ }^{[22]}$ have looked into the potentially differential role of maternal and paternal pain-related appraisals, making it difficult to contextualize the current findings into the broader literature. Other justice-related affectivecognitive processes (e.g., anger) may interact with parental sex; for instance, injustice-related anger may be more pronounced in fathers and therefore more influential to child outcomes ${ }^{[7,32,58]}$. Further, parental injustice appraisals may also differ depending on the sex of their child (as was the case in Study 1), which may be driven by parental expectations for pain tolerance towards boys and girls ${ }^{[41]}$. Future research distinguishing maternal and paternal injustice cognitions and how these may differentially impact sons and daughters is needed.

Perhaps most critically, additional research is necessary to further explicate the phenomenology of parental injustice appraisals in the context of child pain and thus shed light on context-specific content that may not be captured in current conceptualizations of parental pain-related injustice appraisals. While current findings support the criterion validity of the IEQ-Pc and IEQ-Ps, they simultaneously suggest that caution is needed when drawing 
conclusions about their construct validity within the parent-child context. Accordingly, a simple extrapolation of the IEQ by adapting its instructions and item content - as was done in the present study - may be suboptimal. The current findings highlight that further research, including qualitative methodology, is required to define the construct of parental injustice appraisals and to develop valid assessment approaches. Otherwise, research may risk adopting a reified view of injustice appraisal as reflected in the IEQ-Pc and IEQ-Ps, without fully capturing the construct ${ }^{[11]}$ in its specific context. Moreover, measures of parental injustice appraisals should be clearly distinct from other related constructs (e.g., pain catastrophizing $^{[52]}$; pain acceptance ${ }^{[61]}$ ) and outcomes (e.g., pain-related disability, which also entails facing losses due to pain). If not, this may lead to an unwarranted inflation of the explanatory power of pain-related injustice appraisal.

A number of limitations needs to be considered. First, the cross-sectional design of the current study does not allow conclusions regarding the causal relation between parental injustice appraisals and child outcomes. Second, as parent role was not assessed in the clinical sample, differential effects of maternal and paternal injustice could not be examined. It is unclear to what extent findings observed amongst the clinical sample reflect maternal and paternal injustice appraisals or the potential interaction between both ${ }^{[22]}$. Third, parent pain status was not explicitly assessed. Therefore, differences in injustice appraisals among parents with and without chronic pain could not be examined. Such variables Lastly, when working with samples from different countries (i.e., Belgium and the US), cross-cultural differences in the experience and expression of pain and injustice need consideration ${ }^{[2,24,45]}$, as research demonstrates significant cross-cultural differences in self-perceived dysfunction, social expectations, attitudes towards health care and willingness to cope in the context of pain.

Notwithstanding these limitations, the current studies support the importance of parental injustice appraisals in understanding child pain experience. Future research is required to 
further understand the phenomenology of parental injustice appraisals, their impact upon child outcomes as well as potential mechanisms of action.

\section{References}

[1] Agtarap S, Scott W, Warren AM, \& Trost Z: Validation of the Injustice Experience Questionnaire in a heterogeneous trauma sample. Rehabil Psychol 61(3): 336-344, 2016

[2] Brena SF, Sanders SH, Motoyama H: American and Japanese chronic low back pain patients: cross-cultural similarities and differences. Clin J Pain 6(2): 118-124, 1990

[3] Brislin, R. W: Back-translation for cross-cultural research. J Cross Cult Psychol 1(3): 185-216, 1970

[4] Caes L, Vervoort T, Eccleston C, Goubert L: Parents who catastrophize about their child's pain prioritize attempts to control pain. Pain, 153(8): 1695-1701, 2012

[5] Caes L, Vervoort T, Eccleston C, Vandenhende M, Goubert L: Parental catastrophizing about child's pain and its relationship with activity restriction: the mediating role of parental distress. Pain 152(1): 212-222, 2011

[6] Chambers CT, Craig KD, Bennett SM: The impact of maternal behavior on children's pain experiences: An experimental analysis. J Pediatr Psychol 27: 293-301, 2002

[7] Chaplin TM, Cole PM, Zahn-Waxler C: Parental socialization of emotion expression: Gender differences and relations to child adjustment. Emotion 5: 80-88, 2005

[8] Claar RL, Walker LS: Functional assessment of pediatric pain patients: Psychometric properties of the Functional Disability Inventory. Pain 121: 77-84, 2006

[9] Craig KD. The social communication model of pain. Can Psychol 50: 22-32, 2009

[10] Crombez G, Bijttebier P, Eccleston C, Mascagni T, Mertens G, Goubert L, Verstraeten K: The child version of the pain catastrophizing scale (PCS-C): a preliminary validation. Pain 104: 639-646, 2003 
[11] Crombez G, De Paepe AL, Veirman E, Eccleston C, Van Ryckeghem DML: Let's talk about pain catastrophizing measures: An item content analysis. Pain (in submission)

[12] Eccleston C, Crombez G, Scotford A, Clinch J, Connell H: Adolescent chronic pain: Patterns and predictors of emotional distress in adolescents with chronic pain and their parents. Pain 108: $221-229,2004$

[13] Engelen V, Haentjens MM, Detmar SB, Koopman HM, Grootenhuis MA: Health related quality of life of Dutch children: psychometric properties of the PedsQL in the Netherlands. BMC Pediatr 9: 68-75, 2009

[14] Fabrigar LR, Wegener DT, MacCallum RC \& Strahan EJ: Evaluating the use of exploratory factor analysis in psychological research, Psychol methods, 4(3), 272, 1999

[15] Faresjö T \& Faresjö A: To match or not to match in epidemiological studies - Same outcome but less power. Int. J. Environ. Res. Public Health, 7, 325-332, 2010

[16] Figley CR. Compassion fatigue: Psychotherapists' chronic lack of self-care. J Clin Psychol 58: $1433-1441,2002$

[17] Flack F, Stahlschmidt L, Dobe M, Hirschfeld G, Strasser A, Michalak J, Wager J, Zernikow B: Efficacy of adding interoceptive exposure to intensive interdisciplinary treatment for adolescents with chronic pain: a randomized controlled trial. Pain 159(11): 2223-2233, 2018

[18] Goubert L, Craig KD, Vervoort T, Morley S, Sullivan MJL, Williams AC, Cano A, Crombez G: Facing others in pain: the effects of empathy. Pain 118: 285-288, 2005

[19] Goubert L, Eccleston C, Vervoort T, Jordan A, Crombez G: Parental catastrophizing about their child's pain. The parent version of the Pain Catastrophizing Scale (PCS-P): a preliminary validation. Pain 123: 254-263, 2006

[20] Hadjistavropoulos T, Craig KD, Duck S, Cano A, Goubert L, Jackson PL, Vervoort T: A biopsychosocial formulation of pain communication. Psychol Bull 137: 910-939, 2011 
[21] Hayton JC, Allen DG \& Scarpello V: Factor retention decisions in exploratory factor analysis: A tutorial on parallel analysis, Org Res Methods, 7(2), 191-205, 2004

[22] Hechler T, Vervoort T, Hamann M, Tietze AL, Vocks S, Goubert L, Hermann C, Wager J, Blankenburg M, Schroeder S, Zernikow B: Parental catastrophizing about their child's chronic pain: Are mothers and fathers different? Eur J Pain 15: 1-9, 2011

[23] Hillebrandt A, Barclay LJ: Observing others' anger and guilt can make you feel unfairly treated: The interpersonal effects of emotions on justice-related reactions. Soc Just Res 30: $238-269,2017$

[24] Hofstede G: Cultural dimensions in management and planning. Asia Pacific J Management 1: $81-99,1984$

[25] Hu LT, Bentler PM: Cutoff criteria for fit indexes in covariance structure analysis: Conventional criteria versus new alternatives. Struct Equ Modeling 6: 1-55, 1999

[26] $\mathrm{Hu}$ LT, Bentler PM: Fit indices in covariance structure modeling: Sensitivity to underparameterized model misspecification. Psychol methods 3: 424, 1998

[27] Hunfeld JAM, Perquin CW, Duivenvoorden HJ, Hazebroek-Kampschreur A, Passchier J, van Suijlekom-Smit LWA, van der Wouden JC: Chronic pain and its impact on quality of life in adolescents and their families. J Pediatr Psychol 26: 145-153, 2001

[28] Jackson DL, Gillaspy Jr JA, Purc-Stephenson R: Reporting practices in confirmatory factor analysis: An overview and some recommendations. Psychol Methods 14: 6-23, 2009

[29] Jordan A, Eccleston C, Crombez G: Parental functioning in the context of adolescent chronic pain: A review of previously used measures. J Pediatr Psychol 33: 640-659, 2008

[30] Jordan AL, Eccleston C, Osborn M: Being a parent of the adolescent with complex chronic pain: An interpretative phenomenological analysis. Eur J Pain 11: 49-49, 2007

[31] Kennedy L, Dunstan DA: Confirmatory factor analysis of the Injustice Experience Questionnaire in an Australian compensable population. J Occup Rehabil 24: 385-392, 2014 
[32] Kring AM, Gordon AH: Sex Differences in Emotion: Expression, Experience and Physiology. J Pers Soc Psychol 74: 686-703, 1998

[33] Marsh HW, Hocevar D: Application of confirmatory factor analysis to the study of selfconcept: First-and higher order factor models and their invariance across groups. Psychol Bull 97: 562,1985

[34] Miller MM, Scott EL, Trost Z, Hirsh AT: Perceived Injustice Is Associated With Pain and Functional Outcomes in Children and Adolescents With Chronic Pain: A Preliminary Examination. J Pain 17: 1217-1226, 2016

[35] Miller MM, Wuest D, Williams AE, Scott EL, Trost Z, Hirsh AT: Injustice perceptions about pain: Parent-child discordance is associated with worse functional outcomes. Pain online, 1-7, 2018

[36] Mohammadi S, de Boer MJ, Sanderman R, Hagedoorn M: Caregiving demands and caregivers' psychological outcomes: The mediating role of perceived injustice. Clin Rehabil 31: 403-413, 2016

[37] Palermo TM: Impact of recurrent and chronic pain on child and family daily functioning: a critical review of the literature. J Dev Behav Pediatr 21: 58-69, 2000

[38] Palermo TM, Chambers CT: Parent and family factors in pediatric chronic pain and disability: An integrative approach. Pain 119:1-4, 2005

[39] Palermo TM, Eccleston C: Parents of children and adolescents with chronic pain. Pain 146:1517,2009

[40] Perneger TV: What's wrong with Bonferroni corrections, British Med J 316: 1236-1238, 1998

[41] Robinson ME, Riley III JL, Myers CD, Papas RK, Wise EA, Waxenberg LB, Fillingim RB: Gender Role Expectations of Pain: Relationship to Sex Differences in Pain. J Pain 2(5):251257,2001 
[42] Rodero B, Luciano JV, Montero-Marín J, Casanueva B, Palacin JC, Gili M, Lopéz del Hoyo Y, Serrano-Blanco A, Garcia-Campayo J: Perceived injustice in fibromyalgia: psychometric characteristics of the Injustice Experience Questionnaire and relationship with pain catastrophising and pain acceptance. J Psychosom Res 73:86-91, 2012

[43] Romano JM, Jensen MP, Turner JA, Good AB, Hops H: Chronic pain patient-partner interactions: Further support for a behavioral model of chronic pain. Behav Ther 31:415-440, 2000

[44] Russell DW: In search of underlying dimensions: The use (and abuse) of factor analysis in personality and social psychology bulletin, Pers Soc Psychol Bull, 28(12):1629-1646, 2002

[45] Sanders SH, Brena SF, Spier CJ, Beltrutti D, McConnell H, Quintero O: Chronic low back pain patients around the world: cross-cultural similarities and differences. Clin J Pain 8:317323,1992

[46] Satorra A, Bentler PM: A scaled difference chi-square test statistic for moment structure analysis. Psychometrika 66:507-514, 2001

[47] Scott W, Trost Z, Bernier E, Sullivan MJ: Anger differentially mediates the relationship between perceived injustice and chronic pain outcomes. Pain 154:1691-1698, 2013

[48] Shao R, Rupp DE, Skarlicki DP, Jones KS: Employee justice across cultures: A meta-analytic review. J Management 39:263-301, 2013

[49] Simons LG, Conger RD: Linking mother-father differences in parenting to a typology of family parenting styles and adolescent outcomes. J Fam Issues 28:212-241, 2007

[50] Sullivan MJ, Adams H, Horan S, Maher D, Boland D, Gross R: The role of perceived injustice in the experience of chronic pain and disability: scale development and validation. J Occup Rehabil 18:249-261, 2008 
[51] Sullivan MJ, Adams H, Martel MO, Scott W, Wideman T: Catastrophizing and perceived injustice: risk factors for the transition to chronicity after whiplash injury. Spine 36:244-249, 2011

[52] Sullivan MJL, Bishop SR, Pivik J: The Pain Catastrophizing Scale: Development and Validation. Psychol Assess 7:524-532, 1995

[53] Sullivan MJ, Davidson N, Garfinkel B, Siriapaipant N, Scott W: Perceived injustice is associated with heightened pain behavior and disability in individuals with whiplash injuries. Psychol Inj Law 2:238-247, 2009

[54] Sullivan MJ, Scott W, Trost Z: Perceived injustice: a risk factor for problematic pain outcomes. Clin J Pain 28:484-488, 2012

[55] Van Kleef GA: How emotions regulate social life: The emotions as social information (EASI) model. Curr Dir Psychol Sci 18:184-188, 2009

[56] Varni JW, Seid M, Kurtin PS. PedsQL ${ }^{\text {TM }}$ 4.0: Reliability and validity of the Pediatric Quality of Life Inventory ${ }^{\mathrm{TM}}$ Version 4.0 Generic Core Scales in healthy and patient populations. Med Care 39:800-812, 2001

[57] Vervoort T, Goubert L, Eccleston C, Bijttebier P, \& Crombez, G: Catastrophic thinking about pain is independently associated with pain severity, disability, and somatic complaints in school children and children with chronic pain. J pediatr psychol, 31(7), 674-683, 2005

[58] Vervoort T, Huguet A, Verhoeven K, \& Goubert L: Mothers' and fathers' responses to their child's pain moderate the relationship between the child's pain catastrophizing and disability. Pain, 152(4), 786-793, 2011

[59] Vervoort T, Trost Z: Examining affective-motivational dynamics and behavioral implications within the interpersonal context of pain. J Pain 18:1174-1183, 2017

[60] Vervoort T, Trost Z, Sütterlin S, Caes L, Moors A: Emotion regulatory function of parent attention to child pain and associated implications for parental pain control behaviour. Pain $155: 1453-1463,2014$ 
[61] Vowles KE, McCracken LM, McLeod C, Eccleston C: The Chronic Pain Acceptance Questionnaire: confirmatory factor analysis and identification of patient subgroups. Pain 140:284-291, 2008

[62] Walker LS, Greene JW: The functional disability inventory: measuring a neglected dimension of child health status. J Pediatr Psychol 16:39-58, 1991

[63] Weitzner MA, Jacobsen PB, Wagner H, Friedland J, Cox C: The Caregiver Quality of Life Index-Cancer (CQOLC) scale: Development and validation of an instrument to measure quality of life of the family caregiver of patients with cancer. Qual Life Res 8:55-63, 1999

[64] Wheaton B, Muthén B, Alwin D, Summers G. Assessing reliability and stability in panel models. In: Heise DR, editor., Sociological methodology 1977, Jossey-Bass, San Francisco.

[65] Yamada K, Adachi T, Mibu A, Nishigami T, Motoyama Y, Uematsu H, Matsuda Y, Sato H, Hayashi K, Cui R, Takao Y, Shibata M, Iso H: Injustice Experience Questionnaire, Japanese version: cross-cultural factor-structure comparison and demographics associated with perceived injustice. PloS one 11:e0160567, 2016

[66] Zeiss A M, Gallagher-Thompson D, Lovett S, Rose J, McKibbin C: Self-efficacy as a mediator of caregiver coping: Development and testing of an assessment model. J clin Geropsychol 5:221-230, 1999 